\title{
Correlation Between Keratometric and Refractive Astigmatism in Pseudophakes
}

\author{
Shavini Athukorala' \\ Neal Kansara ${ }^{2}$ \\ Erik Lehman ${ }^{3}$ \\ Seth M Pantanelli (iD) \\ 'Department of Ophthalmology, Penn \\ State College of Medicine, Hershey, PA, \\ USA; '2Department of Ophthalmology, \\ Baylor College of Medicine, Houston, \\ TX, USA; ${ }^{3}$ Department of Public Health \\ Sciences, Penn State College of Medicine, \\ Hershey, PA, USA
}

Purpose: To investigate the relationship between measured anterior, posterior, and total keratometric astigmatism and post-operative refractive astigmatism (RA) after cataract surgery.

Patients and Methods: This was a retrospective analysis of eyes that consecutively underwent pre-operative measurements of keratometric astigmatism with a swept-source optical coherence tomography (SS-OCT)-based optical biometer and dual-Scheimpflug/Placido disc corneal topographer, cataract surgery with implantation of a monofocal intraocular lens, and post-operative manifest refraction. The difference between post-operative refractive astigmatism and keratometric astigmatism measured using four different ways [Keratometry (K), Simulated Keratometry (SimK), Total Keratometry (TK), and Total Corneal Power (TCP)] was calculated. Results: For all 118 eyes, a smaller mean vector difference between post-operative refractive astigmatism and measured keratometric astigmatism was realized with TK $(0.08 @ 151)$ vs TCP2 (0.30@ 174; p < 0.0006), as well as with K (0.26@ 173) vs SimK (0.52@ 177; $\mathrm{p}=0.036)$. The mean vector difference between post-operative refractive astigmatism and TK astigmatism was $0.31 @ 097,0.21 @ 163$, and $0.69 @ 179$ in eyes with against-the rule (ATR), oblique, and with-the-rule (WTR) anterior corneal astigmatism, respectively ( $\mathrm{p}<$ 0.0006). On the other hand, posterior corneal astigmatism did not significantly change with the orientation of anterior corneal astigmatism [0.10@180 for ATR, $0.22 @ 180$ for oblique, and $0.28 @ 180$ for WTR $(\mathrm{p}=0.58)]$.

Conclusion: Compared with the other measures of corneal astigmatism, total keratometric astigmatism from the SS-OCT device most closely correlated with post-operative RA. The difference between anterior corneal astigmatism and refractive astigmatism is not completely explained by the contribution from the posterior cornea. Other contributors, such as lens tilt or neuro-adaptation, may be at play.

Keywords: astigmatism, biometry, keratometry

\section{Introduction}

Minimization of refractive error at the time of cataract surgery is an important goal for both surgeons and patients. Since over a third of eyes have clinically significant astigmatism, ${ }^{1}$ correction with limbal relaxing incisions or toric intraocular lenses is often part of the surgical plan. As such, appropriate preparation requires an understanding of the relationship between pre-operative biometry measurements and post-operative refractive astigmatism (RA). The relationship between these two data points is not trivial and is known to include contributions from the anterior and posterior cornea and intraocular media (eg lens tilt); there may also be neuroadaptive or perceptual preferences at work. 
In a previous study, ${ }^{2}$ Kansara et al showed that there was a significant difference between the pre-operative keratometric astigmatism and post-operative RA, even when using total corneal power from a dualScheimpflug-Placido disc corneal tomographer/topographer (Ziemer Galilei G4). Their study dataset did not include total keratometry (TK) from a swept-source OCT (SS-OCT) based biometer (Zeiss IOLMaster 700). We thus sought to determine whether the same findings previously identified with the corneal topographer could be affirmed with an SS-OCT device. The purpose of the present study was to evaluate the agreement between pre-operative anterior and total keratometric astigmatism and post-operative RA in pseudophakic eyes.

\section{Methods}

The study was approved by the Penn State College of Medicine Institutional Review Board (IRB). Given the retrospective nature of the review, the requirement for consent was waived, but the data were anonymized and maintained with confidentiality in accordance with the IRB's Data Management Plan. The study was carried out in compliance with the Declaration of Helsinki. This retrospective analysis of data included eyes that were consecutively evaluated between $11 / 2018$ and $07 / 2020$ that underwent pre-operative biometry (IOLMaster 700), tomography/topography (Galilei G4), cataract surgery with implantation of a monofocal intraocular lens, and manifest refraction 21-90 days post-operatively. Eyes excluded from the analysis included those with measurements deemed to be of poor quality (as defined by the respective devices' image quality metrics), history of ocular surgery or clinically significant corneal disease, cataract surgery combined with another procedure, intraoperative complications, missing post-operative manifest refraction within the time frame of interest, or a best-corrected distance visual acuity worse than 20/40. Post-operative RA was compared to pre-operative astigmatism measured using Keratometry (K; IOLMaster), Simulated Keratometry (SimK; Galilei G4), Total Keratometry (TK; IOLMaster), and Total Corneal Power (TCP2; Galilei G4). An ocular residual astigmatism (ORA) vector was calculated for the four measurement methods, as described by Alpins. ${ }^{3}$ In accordance with best practices as recommended in an editorial by Abulafia et $\mathrm{al}^{4}{ }^{4}$ a multivariate linear model for repeated measures or Hotelling's T-squared test was used to compare posterior corneal astigmatism (PCA) and ORA summated vector mean/centroids, and a generalized
Table I Eye Demographics

\begin{tabular}{|l|l|}
\hline Parameter & $\begin{array}{l}\text { Arithmetic Mean } \pm \text { SD } \\
\text { (Range) }\end{array}$ \\
\hline AL (mm) & $24.45 \pm 1.40(21.78$ to 30.4$)$ \\
\hline Flat K (D) & $43.47 \pm \mathrm{I} .41(40.35$ to 47.17$)$ \\
\hline Steep K (D) & $44.29 \pm 1.40(41.27$ to 48.3$)$ \\
\hline $\begin{array}{l}\text { Anterior keratometric } \\
\text { astigmatism (D) }\end{array}$ & $0.8 I \pm 0.50(0$ to 2.28$)$ \\
\hline $\begin{array}{l}\text { Post-operative manifest refraction } \\
\text { Sphere (D) } \\
\text { Cylinder (D) } \\
\text { SE }\end{array}$ & $-0.65 \pm 0.72(-4.0$ to 0.50$)$ \\
\hline
\end{tabular}

Abbreviations: $\mathrm{AL}$, axial length; $\mathrm{SE}$, spherical equivalent.

estimated equation methodology was used to compare proportions of eyes with ORA vector magnitudes less than 0.5 and 1.0 D. A Bonferroni correction was applied to account for multiple comparisons, with statistical significance assumed at $\mathrm{p}<0.05$.

\section{Results}

One-hundred eighteen eyes met the criteria for inclusion. The relevant biometric parameters describing these eyes are displayed in Table 1. The age of patients ranged from 21 to 90 years old. Fifty-five eyes (47\%) belonged to males and sixty-three (53\%) belonged to females. Fiftyfour (46\%) and sixty-four (54\%) were right and left eyes, respectively. Based upon anterior corneal power measurements from the optical biometer, $50(42 \%), 45(38 \%)$, and $23(20 \%)$ had against-the-rule (ATR), with-the-rule (WTR), and oblique astigmatism, respectively.

The PCA, as measured by the optical biometer, had a steep meridian oriented vertically in $87 \%$ of eyes. PCA arithmetic mean was $0.22 \mathrm{D}$, and the summated vector mean (centroid) was 0.19 @ 180 for all eyes. Broken down by sub-group, the PCA centroid was $0.10 @ 180$, $0.22 @ 180$, and $0.28 @ 180$ for ATR, oblique, and WTR anterior corneal astigmatism. Although the centroid magnitude of PCA slowly increased with increasing WTR astigmatism, this trend was not statistically significant ( $p$ $=0.58)$. In other words, both the arithmetic and vector mean magnitudes of PCA were stable, regardless of anterior corneal astigmatism orientation and magnitude.

The summated vector mean/centroid of ORA vectors for the four measurement methods is displayed in Table 2, and double angle plots depicting the refractive astigmatism 
Table 2 Ocular Residual Astigmatism Centroids by Measurement Method

\begin{tabular}{|l|l|l|l|l|}
\hline \multirow{2}{*}{ Group } & Keratometry (K) & Simulated Keratometry (SimK) & Total Keratometry (TK) & Total Corneal Power (TCP2) \\
\cline { 2 - 5 } & \multicolumn{4}{|c|}{ Centroid \pm SD (D) } \\
\hline All eyes $(n=118)$ & $0.26 @ 173 \pm 0.75^{*}$ & $0.52 @ 177 \pm 0.75^{*}$ & $0.08 @ 151 \pm 0.77^{\dagger}$ & $0.30 @ 174 \pm 0.81^{\dagger}$ \\
ATR eyes $(n=50)$ & $0.17 @ 107 \pm 0.73$ & $0.24 @ 146 \pm 0.78$ & $0.31 @ 097 \pm 0.69$ & $0.16 @ 116 \pm 0.76$ \\
Oblique eyes $(n=23)$ & $0.14 @ 173 \pm 0.53$ & $0.43 @ 180 \pm 0.66$ & $0.21 @ 163 \pm 0.49$ & $0.30 @ 001 \pm 0.72$ \\
WTR eyes $(n=45)$ & $0.77 @ 179 \pm 0.54$ & $0.90 @ 001 \pm 0.55$ & $0.69 @ 179 \pm 0.58$ & $0.86 @ 179 \pm 0.62$ \\
\hline
\end{tabular}

Notes: $* / \dagger$, difference is statistically significant.

Abbreviations: ATR, against-the-rule; WTR, with-the-rule.

estimation error for each eye, using the four different measurement methods, are shown in Figure 1. When all eyes were considered, a smaller ORA summated vector mean/centroid was realized with K vs $\operatorname{SimK}(\mathrm{p}=0.036)$, as well as with TK vs TCP2 $(\mathrm{p}<0.0006)$. The proportion of eyes with ORA magnitudes $<0.5$ were $36.4 \%, 23.7 \%$, $41.5 \%$, and $28.8 \%$ for the four above mentioned measurement methods, respectively, with $\mathrm{K}$ performing similar to $\operatorname{SimK}(p=0.132)$ and TK performing better than TCP2 $(p$ $=0.042)$. The proportion of eyes with ORA magnitudes $<1.0 \mathrm{D}$ were $85.6 \%, 67.8 \%, 83.9 \%$, and $75.4 \%$, respectively ( $p=0.024$ for $K$ vs SimK and $p=0.24$ for TK vs TCP2).

\section{Discussion}

One might prematurely conclude that because the ORA summated vector mean/centroid is statistically equivalent to zero (Hotelling's T-squared $p=0.78$ ) when TK is used, we have successfully accounted for all post-operative RA with pre-operative TK measurements. However, this ORA centroid is the result of averaging data from ATR and WTR sub-groups. When the sub-groups are considered separately, we observe non-zero (Hotelling's T-squared $\mathrm{p}<0.0006)$ ORA centroids of $0.31 @ 097$ in ATR and $0.69 @ 179$ in WTR eyes. In other words, even after all measurable keratometric astigmatism is taken into account, there is an unmistakable amount of unaccounted for astigmatism that increases with the amount of WTR astigmatism in the eye. In fact, we observed that in some individuals with $3 \mathrm{D}$ of total keratometric astigmatism that the unaccounted for magnitude approached 1.0 D. Since the PCA does not significantly increase with increasing WTR astigmatism, but ORA does, we hypothesize that this non-corneal astigmatism is coming from some other source. The trend is present with such systematic predilection that we do not believe it can be explained by intraocular lens tilt or PCA measurement error.
While the present study and that of Kansara et al may be the first to describe these findings in detail, they are in fact well corroborated by recommendations from modern toric intraocular lens calculators [ie Barrett Toric Calculator (BTC)]. ${ }^{5}$ When nominal values of 24 for axial length, $44 @ 180$ for Flat K, $45.5 @ 90$ for Steep K, 3 for anterior chamber depth, 4.5 for lens thickness, and 12 for white-to-white are entered, the calculator reveals a "Net Astigmatism" of 0.85 D @ 090, suggesting 0.65 D of astigmatism that is unaccounted for by the 1.5 $\mathrm{D}$ contributed by the anterior corneal surface. When the Steep K magnitude is increased to 47, the BTC reports a "Net Astigmatism" of 2.06 D @ 090, suggesting 0.96 $\mathrm{D}$ of astigmatism unaccounted for by the $3 \mathrm{D}$ contributed by the anterior corneal surface.

It has been previously postulated that neuro-adaptation is a contributor toward the non-zero ORA. The current study further potentiates this hypothesis: an astigmatic lens contributes power 90 degrees away from its axis, and eyes with pre-operative ATR astigmatism preferred additional focusing power along the horizontal meridian post-operatively; those with WTR astigmatism preferred the opposite. In other words, each sub-group preferred to be driven toward a refractive state similar to that present pre-operatively. The fact that the WTR sub-group had a higher ORA vector magnitude than the ATR group might be explained by the fact that we spend most of our lives with WTR astigmatism, making those neuro-adaptive preferences the strongest.

One limitation of the present study was the inability to account for surgically induced astigmatism (SIA), since post-operative keratometry measurements were not available. To overcome this shortcoming, we attempted to empirically account for SIA by adding varying amounts of astigmatism (between 0.01 and 0.25D) 90 degrees away from the incision axis, but every empirical model resulted in increases in ORA 


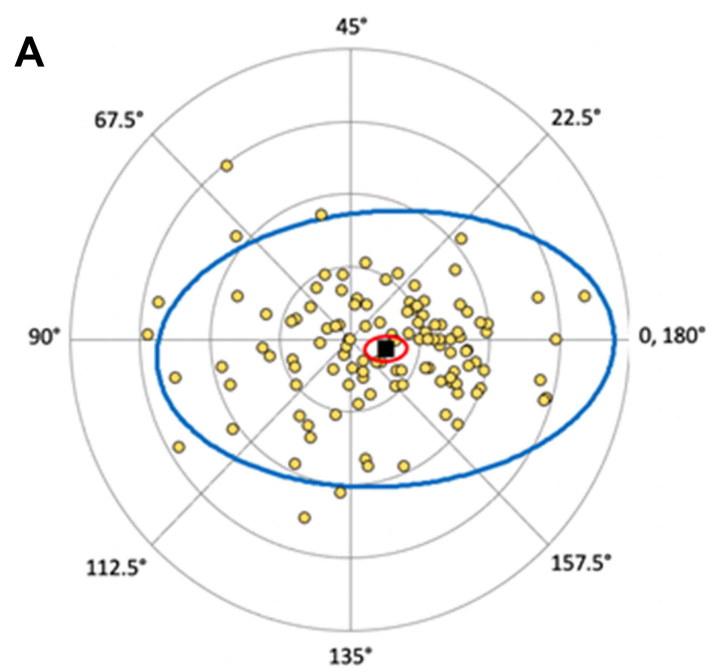

Centroid : 0.26D @ 173ะ0.76D

Mean Absolute: $\quad 0.690 \pm 0.41 D$

$\mathrm{N}=118$

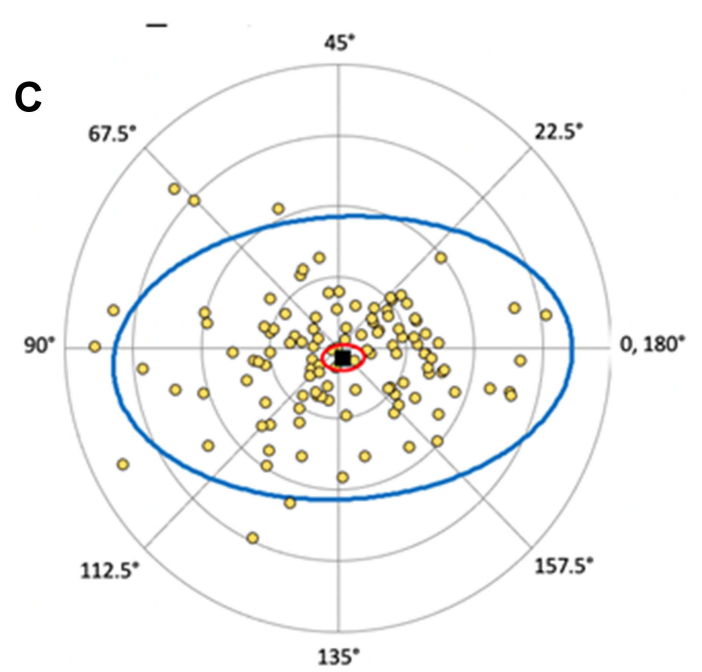

Centroid : 0.08D @ 148 $\pm 0.78 D$

Mean Absolute: $\quad 0.66 D \pm 0.43 D$

$N=118$

\section{Centroid}

\section{5\% confidence ellipse of the centroid}

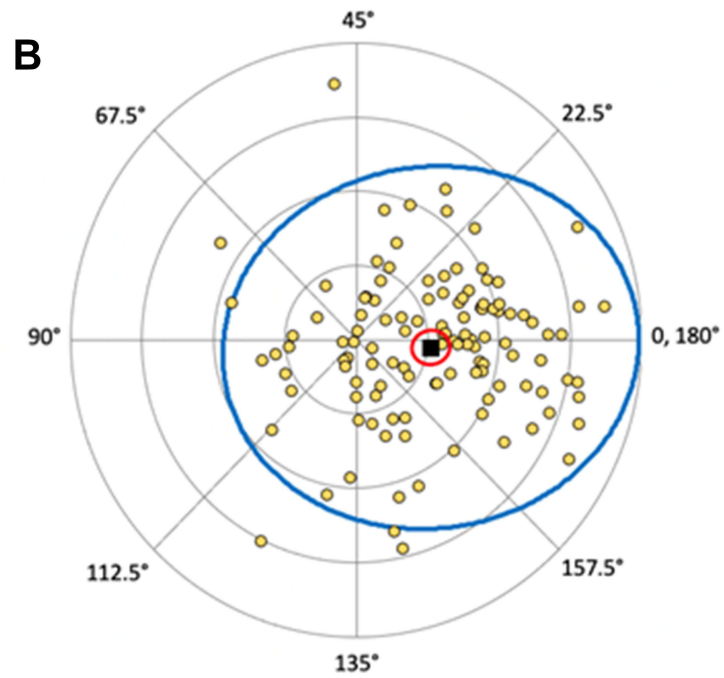

Centroid : 0.52D@ 177 $0.76 D$

Mean Absolute: $\quad 0.82 D \pm 0.42 D$

$N=118$

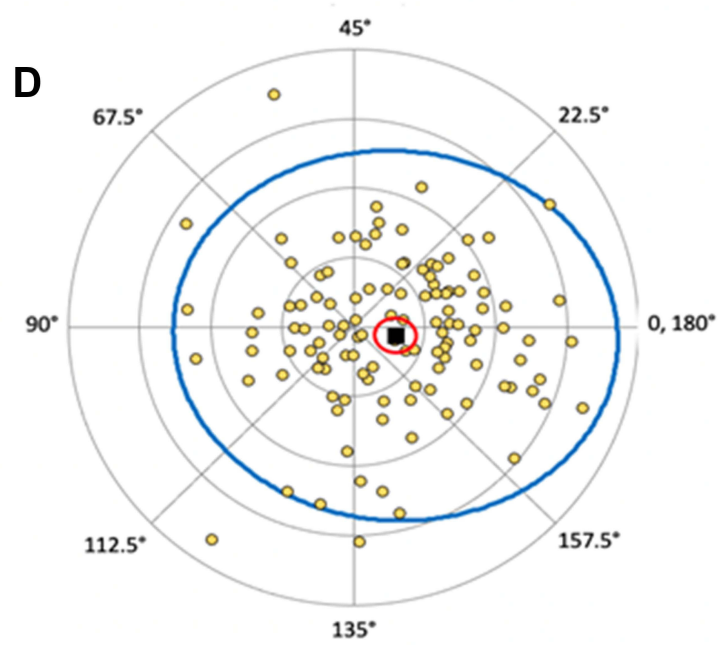

Centroid : 0.30D @ 174 $\pm 0.82 D$

Mean Absolute:

$0.76 D \pm 0.43 D$

$N=118$

Each ring $=0.50 \mathrm{D}$

\section{5\% confidence ellipse of the dataset}

Figure I Double-angle plots depicting ocular residual astigmatism (ORA) using (A) Keratometry; K, (B) Simulated Keratometry; SimK, (C) Total Keratometry; TK, and (D) Total Corneal Power; TCP.

vector magnitudes (eg attempts to incorporate SIA worsened the modeling). This is not completely surprising - in fact, it is recommended to use an SIA of zero when using the Kane toric calculator, ${ }^{6}$ and Warren Hill suggests that SIA likely resides between zero and $0.1 \mathrm{D}$ for clear corneal incisions less than $2.75 \mathrm{~mm}$ (personal communication). Furthermore, the
IOLMaster 700's User Manual suggests that the SDs and repeatability limits for cylinder measurements are 0.148 and 0.414 , respectively. Since these values are higher than the $0-0.1 \mathrm{D}$ effect known to come from SIA, the device may not be sensitive enough to accurately take into account its effect. ${ }^{7}$ Nevertheless, any small amount of unaccounted for SIA is unlikely to 
explain the large ORA magnitudes with opposing subgroup orientations.

\section{Conclusion}

The present work demonstrates that SS-OCT keratometry most closely correlates with post-operative RA when compared with other measurement methods; however, there are still additional factors contributing to RA beyond the anterior and posterior cornea. These findings, previously identified using anterior and posterior corneal curvature measurements from Scheimpflug images, have now been confirmed by the present study using a second imaging modality, SS-OCT. Further work is needed to investigate whether these factors might be directly measurable or more accurately predicted at the individual level.

\section{Funding}

This study was funded in part by an investigator initiated trial grant from Ziemer Ophthalmic Systems AG, and by the National Center for Advancing Translational Sciences, National Institutes of Health (NIH), through Grant UL1 TR002014 and Grant UL1 TR00045. The content is solely the responsibility of the authors and does not necessarily represent the official views of the NIH, Bethesda, Maryland, USA.

\section{Disclosure}

SMP serves as an advisory board member and consultant for Carl Zeiss Meditec, and as a consultant for
Bausch \& Lomb. He also reports lecture fees/honoraria from Alcon, outside the submitted work. NK reports travel reimbursement to a wet lab course in St Louis from Bausch and Lomb, outside the submitted work. EL reports grants from NIH CTSI, during the conduct of the study. The authors report no other conflicts of interest in this work.

\section{References}

1. Hoffer KJ. Biometry of 7,500 cataractous eyes. Am J Ophthalmol. 1980;90:360-368. doi:10.1016/S0002-9394(14)74917-7

2. Kansara N, Cui D, Banerjee K, Landis Z, Scott IU, Pantanelli SM. Anterior, posterior, and non-keratometric contributions to refractive astigmatism in pseudophakes. J Cataract Refract Surg. 2020;47 (1):93-99. doi:10.1097/j.jcrs.0000000000000390

3. Alpins NA. New method of targeting vectors to treat astigmatism. J Cataract Refract Surg. 1997;23(1):65-75. doi:10.1016/S08863350(97)80153-8

4. Abulafia A, Koch DD, Holladay JT, Wang L, Hill W. Pursuing perfection in intraocular lens calculations: IV. Rethinking astigmatism analysis for intraocular lens-based surgery: suggested terminology, analysis, and standards for outcome reports. J Cataract Refract Surg. 2018;44(10):1169-1174. doi:10.1016/j.jcrs.2018.07.027

5. Barrett G. Barrett toric calculator. San Diego, CA: ASCRS. Available from: https://ascrs.org/tools/barrett-toric-calculator. Accessed July 23, 2021.

6. Kane JX. Kane formula. Available from: https://www.iolformula.com/ about/. Accessed July 20, 2021.

7. Carl Zeiss Meditec AG. IOLMaster 700: software release 1.90, instructions for use. Jena, Germany; 2020.
Clinical Ophthalmology

\section{Publish your work in this journal}

Clinical Ophthalmology is an international, peer-reviewed journal covering all subspecialties within ophthalmology. Key topics include: Optometry; Visual science; Pharmacology and drug therapy in eye diseases; Basic Sciences; Primary and Secondary eye care; Patient Safety and Quality of Care Improvements. This journal is indexed on PubMed

Submit your manuscript here: https://www.dovepress.com/clinical-ophthalmology-journal

\section{Dovepress}

Central and CAS, and is the official journal of The Society of Clinical Ophthalmology (SCO). The manuscript management system is completely online and includes a very quick and fair peer-review system, which is all easy to use. Visit http://www.dovepress.com/ testimonials.php to read real quotes from published authors. 